\title{
Neurosyphilis with optical involvement in an immunocompetent patient: a case report
}

This article was published in the following Dove Press journal:

International Medical Case Reports Journal

14 February 2012

Number of times this article has been viewed

\section{Rui Paulo Rodrigues \\ Nuno Correia \\ António Vieira Lopes}

Internal Medicine Service, Saint John Hospital Center, Porto, Portugal
Correspondence: Rui Paulo Rodrigues Internal Medicine Service, Centro Hospitalar de São João, Hospital de São João Alameda Professor Hernâni Monteiro, 4200-319 Porto, Portugal

Tel +35 I 964668669

$\mathrm{Fax}+35$ I 2255I2332

Email rppr.med@gmail.com
Background: Neurosyphilis became a rare disease after the introduction of antibiotics. The resurgence of syphilis in association with human immunodeficiency virus infection in the past decades increased the incidence of this tertiary form of the disease. However, in immunocompetent patients neurosyphilis remains uncommon and accurate diagnosis is challenging as the clinical presentation is unspecific and the validity of supportive laboratory tests is unclear.

Case report: We describe the case of a 60-year-old man who presented with a 6-month clinical picture of depression-like symptoms and recent sudden unilateral blindness. Medical history uncovered a primary syphilitic event 20 years before. The investigation led to the diagnosis of neurosyphilis with optical involvement in a patient who did not present with any signs of secondary syphilis. Treatment with penicillin $G$ and topical steroids resulted in significant clinical improvement and resolution of the visual loss.

Conclusion: In the well-established natural history of syphilis, primary syphilis is almost always followed by manifestation of the secondary form, which in turn precedes a period of latency. This case emphasizes the need for a high index of suspicion of neurosyphilis in an immunocompetent patient with nonspecific neuropsychiatric manifestations and an atypical course of syphilis infection.

Keywords: syphilis, neurosyphilis, parenchymatous neurosyphilis

\section{Introduction}

Syphilis is a sexually transmitted disease caused by Treponema pallidum. Antibiotic treatment has reduced the incidence of syphilis over the past decades to the extent that it is now considered a rare condition, thus occasionally misdiagnosed. ${ }^{1}$ Importantly, the widespread use of antibiotics led to changes in its classic clinical presentation. In addition, the validity of diagnostic laboratory tests is unclear. ${ }^{2}$

Epidemiological data indicate a reemergence of the disease in the last two decades in Europe and the USA. In 1999, the World Health Organization estimated that nearly 12 million new cases of syphilis occurred among adults worldwide. ${ }^{3,4}$ Demographic and socioeconomic shifts such as rising migration from high-prevalence countries and increases in prostitution, men who have sex with men, promiscuity, and underprivilege have been proposed as key factors in the reappearance of the disease. ${ }^{1,5-8}$ Importantly, it has reemerged concurrently with the global epidemic of human immunodeficiency virus (HIV) infection. Nevertheless, syphilis remains underestimated and under-recognized due to the variability of clinical presentation and aging of the population with chronic comorbidities. Hence some manifestations of the past are nowadays superimposed on the course of chronic diseases. ${ }^{9}$ 
The evolution of syphilis is classically divided into three symptomatic stages (primary, secondary, and tertiary) and two asymptomatic stages (early and late latent). Primary syphilis is almost invariably followed by manifestations of secondary syphilis, which, in turn, precede a period of latency. In the period of early latent syphilis (up to 1 year after contact) relapses of secondary syphilis can occur in $25 \%$ of cases, but after this period no recurrence develops. The evolution of late latent syphilis ( 1 year after contact) is governed by the classical rule of thirds. In one-third of cases there are no manifestations of disease and nontreponemal serological reactions (Venereal Disease Research Laboratory [VDRL]) of syphilis become VDRL negative. In another third, VDRL remains positive, usually with low titers, but also without any signs or symptoms of syphilis. In the last third, late-benign or tertiary syphilis occurs. About half of these patients have late-benign syphilis and the others have cardiovascular or neurological disease. ${ }^{3}$

Syphilitic infection of the nervous system - neurosyphilis - results in the most chronic, insidious meningeal inflammatory process known. ${ }^{4}$ In the pre-antibiotic era it was estimated to account for $10 \%-20 \%$ of asylum admissions. ${ }^{10}$ After the introduction of penicillin, it became rare. Following the epidemiological data of syphilis, neurosyphilis has also increased in patients coinfected with HIV. However, it remains uncommon in non-HIV patients. ${ }^{11,12}$ The clinical picture of neurosyphilis is nonspecific and may manifest itself at any time of the natural history of disease. In HIV patients, it has been reported that the disease follows a more fulminant course. In immunocompetent individuals, the disease manifests more insidiously, with nonspecific symptoms, making the diagnosis more difficult. ${ }^{2}$

We present the case of an immunocompetent patient that sought medical attention due to sudden unilateral blindness and progressive neuropsychiatric complaints. In-hospital investigation established the diagnosis of neurosyphilis with optical involvement. The natural history, investigation, treatment, and follow-up for neurosyphilis are discussed.

\section{Case report}

A 60-year-old man was referred to the emergency department with a 6-month history of asthenia, insomnia, depression, anxiety, and loss of libido. He had been medicated with a benzodiazepine with no improvement. The last 2 months were marked by an overall decline in memory, recurrent and very intense headaches with unilateral ocular pain, and sudden right eye visual loss. Simultaneously, the patient complained of widespread rash. He had no other complaints and at first he denied history of sexual risk.

His medical history was remarkable for recurrent stroke events (the first episode had occurred 17 years ago), ischemic heart disease, hypertension, and dyslipidemia.

His usual medication included: acetylsalicylic acid, clopidogrel, isosorbide mononitrate, ramipril, bisoprolol, pravastatin, and clorazepate dipotassium. He denied any known adverse drug reactions.

Physical examination documented a generalized erythematous rash with scaly small patches predominantly affecting the face and extremities (Figure 1). The neurological examination was remarkable for cognitive dysfunction. Motor, sensory, and cerebellar tests were normal. The mini-mental status examination score was 27/30. He had no temporal artery tenderness or decreased temporal artery pulse. No Argyll Robertson pupils were observed. Right eye fundus examination revealed an incipient papilledema. Visual acuity was measured as less than 20/400 (counting fingers classification). Color vision was not affected. The remaining physical examination was otherwise normal.

Initial blood tests showed hemoglobin $13.9 \mathrm{~g} / \mathrm{dL}$, white blood cell (WBC) count of $7780 \mathrm{WBC} / \mu \mathrm{L}$ (71\% neutrophils and $23 \%$ lymphocytes), C-reactive protein $3.7 \mathrm{mg} / \mathrm{L}$ (normal $<3 \mathrm{mg} / \mathrm{L}$ ), and an erythrocyte sedimentation rate of $22 \mathrm{~mm} / \mathrm{hr}$, with no additional relevant findings. A brain computerized tomography scan excluded acute lesions (Figure 2).

The patient was admitted to the internal medicine service for further management. Initial investigation for a possible infectious etiology in the differential diagnosis of the neuropsychiatric abnormalities revealed serum reactivity to the T. pallidum particle agglutination (TPPA) test (Table 1). The patient then confirmed the appearance of a dorsal painful penile lesion, approximately 20 years ago, which had occurred some weeks after sexual risk intercourse and spontaneously resolved 4 weeks later.

Cerebral spinal fluid (CSF) analysis revealed: $15 \mathrm{WBC} / \mu \mathrm{L}$ (11 lymphocytes $/ \mu \mathrm{L}$ ) glucose $0.65 \mathrm{~g} / \mathrm{L}$ ( $69 \%$ of serum), total protein $0.97 \mathrm{~g} / \mathrm{L}$; a positive fluorescent treponemal antibody absorption (FTA/Abs) test, and negative VDRL test.

Additional tests ruled out infection by other agents and the remaining blood analysis was unrevealing. The result from the skin lesions biopsy was nonspecific.

The patient was treated for 2 weeks with penicillin $G$ (4 million units intravenously every 4 hours) and topical ophthalmic corticosteroid. Vision recovery (20/30, without 

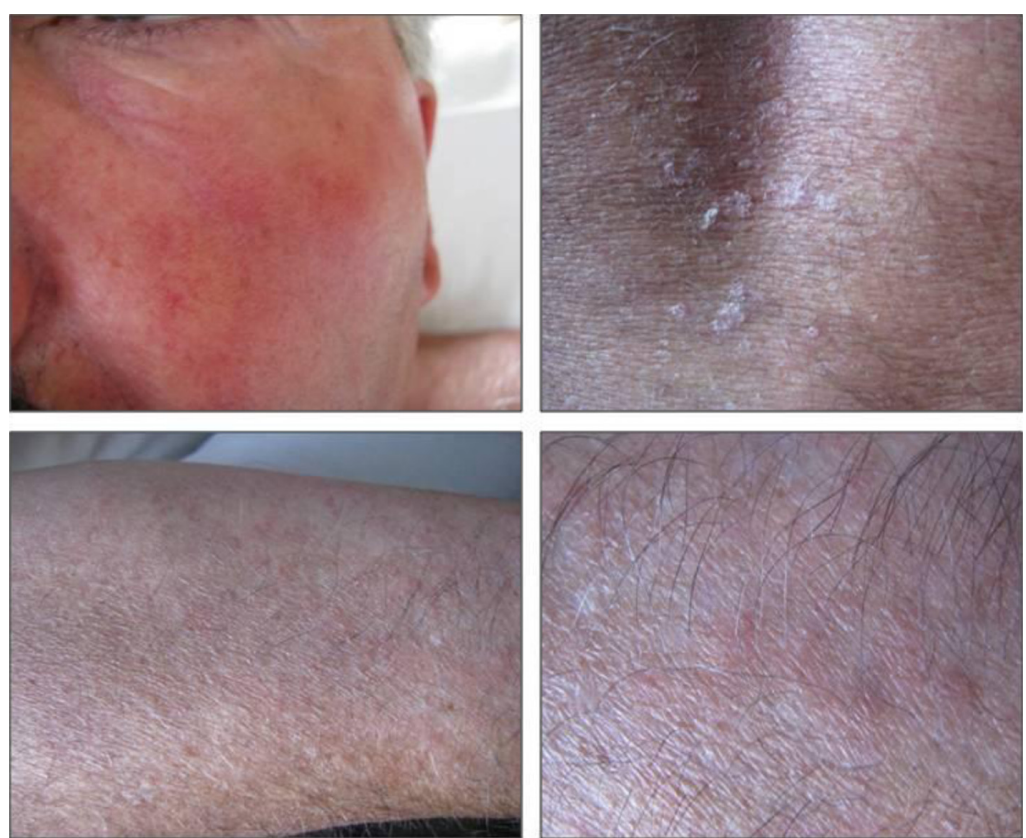

Figure I Generalized erythematous rash with scaly small patches predominantly affecting the face and extremities.

papilledema) was documented after 1 week and significant neuropsychiatric improvement occurred after completion of the treatment. As the skin rash did not improve after treatment, it was not considered a syphilis manifestation.
Six months later the patient remained asymptomatic, with no visual complaints and showing improved performances. A lumbar puncture was performed at this time and normalization of CSF cytochemical characteristics was documented (Table 1), excluding the need for further syphilis treatment.

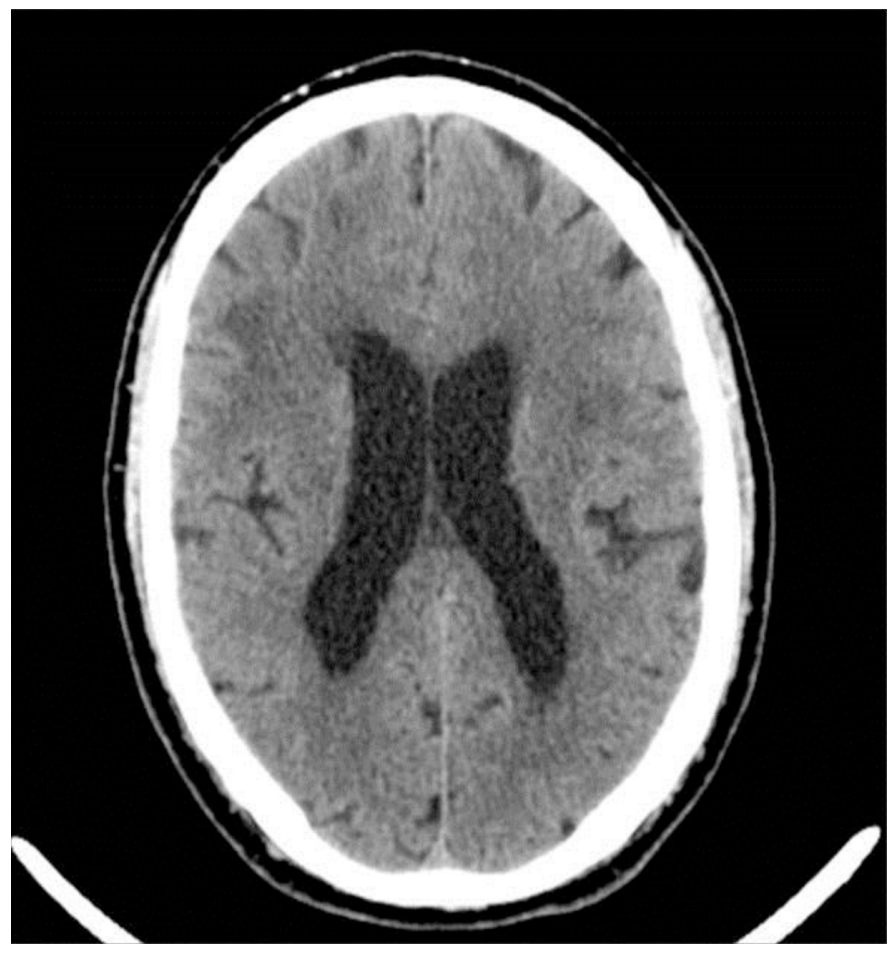

Figure 2 Computed tomography brain scan revealed ischemic leukoencephalopathy as evidenced by multiple hypodensities involving the left basal ganglia, right internal capsule, and bilateral frontal white matter consistent with areas of chronic infarcts. 
Table I Laboratory results

\begin{tabular}{|c|c|}
\hline Sample & Relevant results \\
\hline \multirow[t]{5}{*}{ Blood } & VDRL: reactive \\
\hline & TPPA: reactive \\
\hline & Blood cultures: negative \\
\hline & HIV, HBV, HCV serological markers: \\
\hline & negative for active infection \\
\hline \multirow[t]{9}{*}{ CSF at baseline } & WBC: $15 / \mu \mathrm{L}$ \\
\hline & Glucose: $0.65 \mathrm{~g} / \mathrm{L}$ ( $69 \%$ of serum glucose) \\
\hline & Total protein: $0.97 \mathrm{~g} / \mathrm{L}$ \\
\hline & FTA/Abs: reactive \\
\hline & VDRL: nonreactive \\
\hline & Borrelia burgdoferi serology: negative \\
\hline & Bacterial and mycobacterial cultures: \\
\hline & $\begin{array}{l}\text { negatives } \\
\text { PCR of Mycobacterium tuberculosis and }\end{array}$ \\
\hline & Borrelia burgdorferi: negative \\
\hline CSF 6 months after & WBC: $0 / \mu \mathrm{L}$ \\
\hline \multirow[t]{2}{*}{ treatment } & Total protein $<0,10 \mathrm{~g} / \mathrm{dL}$ \\
\hline & VDRL: nonreactive \\
\hline Skin lesion biopsy & Inconclusive \\
\hline
\end{tabular}

Abbreviations: CSF, cerebral spinal fluid; HBV, hepatitis B virus; HIV, human immunodeficiency virus; $\mathrm{HCV}$, hepatits $\mathrm{C}$ virus; FTA/Abs, fluorescent treponemal antibody absorption; PCR, polymerase chain reaction; TPPA, Treponema pallidum particle agglutination test; VDRL, Venereal Disease Research Laboratory; WBC, white blood cells.

\section{Discussion}

T. pallidum infection usually follows a typical course, with periods of active and latent disease (Figure 3). ${ }^{3,13}$ It is classified as congenital or acquired, usually acquired through sexual intercourse. The European Centre for Disease Prevention and Control additionally classifies installation of early syphilis under 1 year and late installation over and above 1 year. $^{14}$

Characteristically, it follows an incubation period of 10-90 days before the development of an ulcerated lesion recognized as typical of primary syphilis. ${ }^{4}$ This lesion is usually single, painless, with a hard and clean base and usually located in the anogenital region. Any anogenital ulcer should be considered syphilis until proven otherwise. ${ }^{14}$ The primary lesion heals in 2-6 weeks without treatment. ${ }^{4}$ Occasionally, especially in patients coinfected with HIV, the lesion may present atypically - painful, multiple, purulent, destructive, and extra-genital. ${ }^{14}$ The penile lesion described by this patient was highly suggestive of a syphilitic ulcer since it occurred after a proper incubation period and resolved spontaneously.

Following a primary syphilis event, manifestations of secondary syphilis with multiorgan involvement commonly develop. Subsequently, there is a latency period in which serological tests are positive but no symptoms suggestive of infection are perceived. ${ }^{14}$

The history of this patient discussed here suggests an atypical course since a period of latent syphilis developed after a remote primary infection that was not followed by any signs of a possible secondary syphilis stage (Figure 3 ).

The evolution of late latent syphilis is variable and only one-third of the cases evolve to tertiary syphilis. ${ }^{3}$ Tertiary syphilis can be gummatous with clinical manifestations of space-occupying lesions, cardiovascular disease or neurosyphilis, reaching the ocular system, auricular, meningovascular, parenchymal, or even asymptomatic but with CSF abnormalities. $^{3}$

In the early stages of neurosyphilis, up to 5-12 years after primary infection, the pathological changes are limited to the perivascular infiltration of the meninges with lymphocytes and plasma cells - meningovascular syphilis. This condition implies arteritis and can lead to luminal occlusion due to thrombosis, ischemia, and infarction.

Parenchymal involvement occurs 18-25 years after primary infection and is characterized by degeneration, neuronal

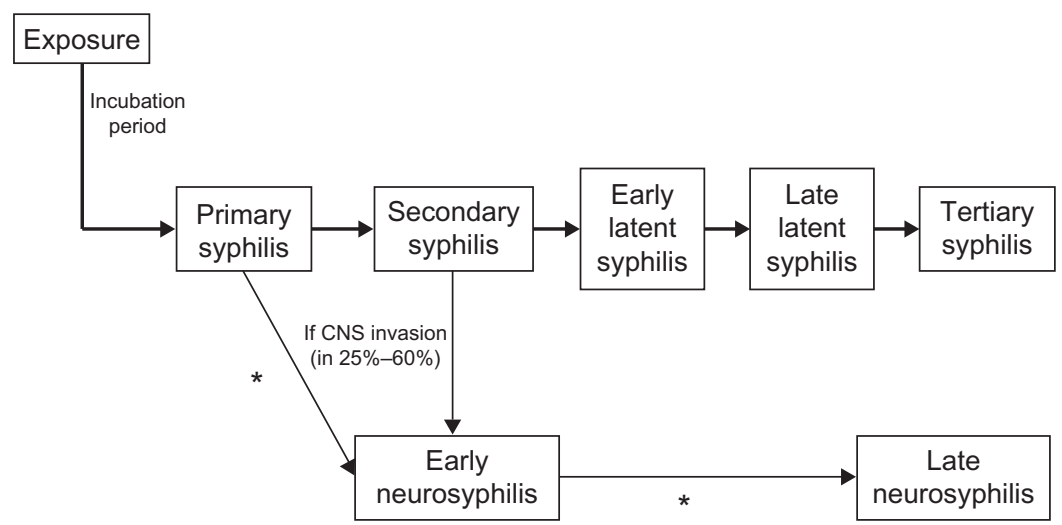

Figure 3 Natural history of untreated syphilis in immunocompetent individual.

Note: *Patient's clinical course in the presented case.

Abbreviation: CNS, central nervous system. 
loss, and gliosis. ${ }^{3,11,15}$ The treatment halts the progression, but recovery of neurological functions rarely occurs. ${ }^{3}$

In young adults without cardiovascular risk factors and with a clinical picture compatible with cerebral ischemia, the possibility of meningovascular syphilis must always be considered. ${ }^{3,11,15}$ This patient had apparently suffered four strokes between the ages of 43 and 50 years old. We were unable to gather any clinically relevant information from those hospitalizations at another institution. One can only speculate about the possibility of meningovascular neurosyphilis as a possible background cause for neurovascular events.

Ocular symptoms in syphilis are highly suggestive of neurological involvement and underlying neurosyphilis is very likely. ${ }^{4,11}$ Neurosyphilis with optic involvement is well described in the literature. Any patient with unexplained loss of vision should be screened for syphilis., ${ }^{9,14}$ Ocular involvement can occur at any time during the course of the disease. It may be asymptomatic or present as an anterior uveitis, interstitial keratitis, retinal vasculitis, retinitis, optic neuritis, dacryoadenitis, or scleritis. ${ }^{1,11}$ Optic nerve implication may be unilateral or bilateral and manifest as perineuritis, optic neuritis, and papilledema with loss of vision. ${ }^{4}$ The classic Argyll Robertson pupil occurs in $48 \%-64 \%$ of patients with late parenchymal neurosyphilis. ${ }^{16}$

The diagnosis of neurosyphilis is based on clinical history, physical examination, and laboratory studies. ${ }^{3,11}$ The screening should preferentially use an antigen treponemal test - T. pallidum hemagglutination assay (TPHA), TPPA, FTA/Abs test, treponemal enzyme immunoassay. Rapid plasma reagin (RPR) tests or VDRL, both nontreponemal antigen, are not recommended as screening tests. ${ }^{14}$ After a positive screening test, the infection must be confirmed by another treponemal antigen test. The FTA/Abs test is not recommended as a standard confirmatory test, as it depends on highly specialized laboratories, where the quality of reagents and reproducibility of the test can be assured..$^{14}$
The quantification of VDRL or RPR is recommended when there is confirmation of diagnosis and is useful for monitoring the activity of infection and response to treatment. Active disease with negative serum VDRL or RPR is a rare situation. ${ }^{14}$

CSF analysis should be performed in all patients with positive syphilitic serological tests and neurological, ocular, or auditory manifestations, treatment failure, or concomitant HIV infection. ${ }^{3,14} \mathrm{CSF}$ abnormalities of active neurosyphilis include: pleocytosis with $10-100 \mathrm{WBC} / \mu \mathrm{L}$, increased number of mononuclear cells $(>5-10$ cells $/ \mu \mathrm{L})$, increased protein between $0.50 \mathrm{~g} / \mathrm{L}$ and $1.0 \mathrm{~g} / \mathrm{L}$, positive VDRL ( $90 \%$ of cases), and decreased glucose concentration ( $45 \%$ of the cases). ${ }^{3}$ Our hospital laboratory is not able to provide the differential count of CSF WBCs whenever the cell count is $<50-100$ cells $/ \mu \mathrm{L}$, due to lack of sensitivity of the available technique. However, CSF pleocytosis was observed in our patient and absence of the differential count does not rule out neurosyphilis. ${ }^{14}$

Testing for treponemal and nontreponemal reactions in the CSF is the most sensitive and specific laboratory method for diagnosis of neurosyphilis. The presence of positive CSF VDRL confirms the diagnosis. However, a nonreactive VDRL test does not exclude it. ${ }^{3,13}$ Like all serological tests, regardless of the disease, the existence of false positives or false negatives must be considered (Table 2).

Despite these available laboratory tests, it is worth noting that no single test or clinical feature can diagnose neurosyphilis and a diagnosis is usually made based on a combination of clinical presentation and laboratory tests. ${ }^{14}$ Nevertheless, the laboratory results, according to the literature, are sufficient to establish the diagnosis of neurosyphilis in the presented case (Table 3).

The use of penicillin is the recommended treatment for all stages of syphilis. In cases of ocular involvement, the use of topical steroids should also be considered. ${ }^{2,11}$ The recommended treatment for patients not allergic to penicillin is the administration of 18-24 million units per day of the

Table 2 Considerations regarding the validity of syphilis serological blood tests according to the European Centre for Disease Prevention and Control ${ }^{14}$

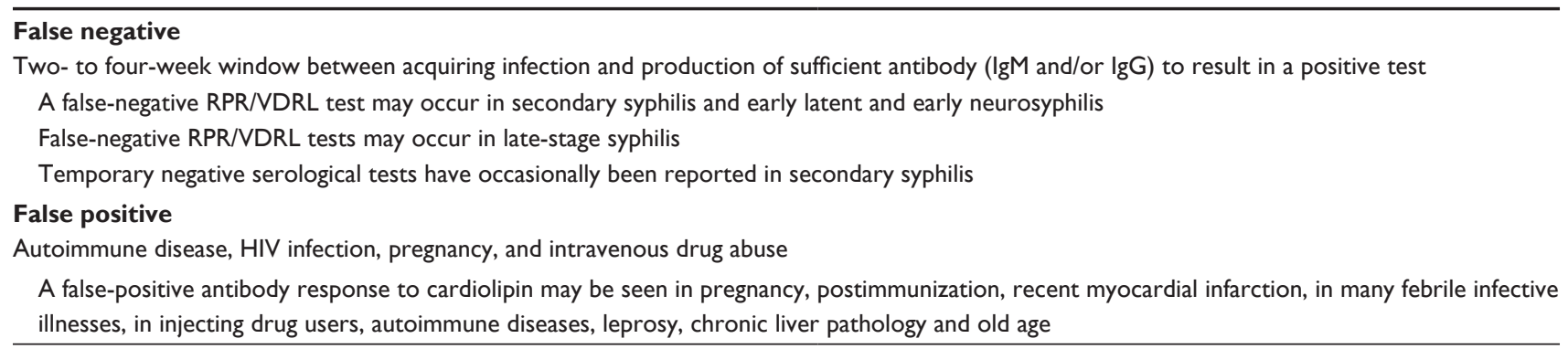

Abbreviations: HIV, human immunodeficiency virus; Ig, immunoglobulin; RPR, rapid plasma reagin test; VDRL, Venereal Disease Research Laboratory. 
Table 3 Diagnostic criteria comparison according to European and US guidelines ${ }^{6,14,18}$

\begin{tabular}{|c|c|}
\hline $\begin{array}{l}\text { IUSTI: } 2008 \text { European guidelines } \\
\text { on the management of syphilis }\end{array}$ & CDC: Sexually transmitted diseases treatment guidelines, 2010 \\
\hline $\begin{array}{l}\text { Criteria for the diagnosis of neurosyphilis in CSF: } \\
\text { - TPHA/TPPA and/or FTA-abs test positive } \\
\text { and } \\
\text { - Increased number of mononuclear cells ( } 5-10 \text { cells } / \mu \mathrm{L}) \\
\text { or positive VDRL/RPR }\end{array}$ & $\begin{array}{l}\text { - CNS or ophthalmic signs or symptoms } \\
\text { plus } \\
\text { - Serologic evidence (positive nontreponemal and treponemal test results) } \\
\text { for syphilis infection } \\
\text { Plus one of the following: } \\
\text { - Positive VDRL-CSF } \\
\text { - Increased CSF protein ( }>40 \mathrm{mg} / \mathrm{dL}) \\
\text { - Increased CSF WBC count }(>5 \text { mononuclear cells } / \mu \mathrm{L})\end{array}$ \\
\hline
\end{tabular}

Abbreviations: CDC, Centers for Disease Control and Prevention; CNS, central nervous system; CSF, cerebral spinal fluid; FTA/Abs, fluorescent treponemal antibody absorption; IUSTI, International Union Against Sexually Transmitted Infection; RPR, rapid plasma regain; TPHA, Treponema pallidum hemagglutination assay; TPPA, T. pallidum particle agglutination test; VDRL, Venereal Disease Research Laboratory; WBC, white blood cell.

aqueous crystallized penicillin $\mathrm{G}$ intravenously in fractionated doses of 3-4 million U 4-hourly for 10-14 days. . $^{1,31,14-17}$ In penicillin-allergic patients, desensitization to penicillin or use of erythromycin or ceftriaxone should be considered. ${ }^{14}$

Ocular syphilis should be treated like any other form of neurosyphilis. Antibiotics in combination with topical steroids should be considered. ${ }^{1,11}$ Usually there is a good therapeutic response, which is not affected by the presence of HIV infection. ${ }^{1,3,11,14,17}$

Lumbar puncture must be repeated every 6 months until cell counts normalize. A new course of therapy should be considered if CSF features remain abnormal. Specific tests for T. pallidum remain positive even after treatment. ${ }^{1,3,9,11,14}$

\section{Conclusion}

Syphilis remains a great imitator because its clinical expression is not pathognomonic and it may be misdiagnosed when superimposed on chronic diseases. Neurosyphilis, particularly its atypical forms characterized by nonspecific neuropsychiatric symptoms, remains a diagnostic challenge. The definitive diagnosis relies on the correct interpretation of laboratory tests of peripheral blood and CSF, in addition to a suggestive clinical history.

This case illustrates the importance of keeping a high grade of suspicion of neurosyphilis in immunocompetent patients with ocular and neuropsychiatric complaints since its nonrecognition may have serious and irreversible consequences. Depending on the baseline neurological status, early and adequate treatment with penicillin and steroids may restore eye integrity and prevent serious damaging of the central nervous system.

\section{Authors' contributions}

All authors were involved in the approach and management of the presented case. Rui Paulo Rodrigues made substantial contributions in gathering patient data and performed an extensive up-to-date review of the literature. Nuno Correia was involved in conception and design of the case as well as English writing. Rui Paulo Rodrigues and Nuno Correia were equally involved in drafting the manuscript and revising it critically. António Vieira Lopes was responsible for final approval of the version to be published and made intellectually important contributions.

\section{Acknowledgments}

Written informed consent was obtained from the patient for publication of this case report and accompanying images. A copy of the written consent is available for review from the Editor-in-Chief of this journal.

\section{Disclosure}

The authors report no conflicts of interest in this work. No grant support or other kind of assistance was obtained.

\section{References}

1. Prokosch V, Thanos S. Emerging syphilitic optic neuropathy: critical review and recommendations. Restor Neurol Neurosci. 2008; 26(4-5):279-289.

2. Timmermans M, Carr J. Neurosyphilis in the modern era. J Neurol Neurosurg Psychiatry. 2004;75(12):1727-1730.

3. Barros RC, Lisboa A, Sá C, Resende M. Neurossífilis - Revisão Clínica e Laboratorial [neurosyphilis - clinical and laboratorial review]. Arq Med. 2005;19(3):121-129. Portuguese.

4. Smith GT, Goldmeier D, Migdal C. Neurosyphilis with optic neuritis: an update. Postgrad Med J. 2006;82(963):36-39.

5. Chang CC, Leslie DE, Spelman D, et al. Symptomatic and asymptomatic early neurosyphilis in HIV-infected men who have sex with men: a retrospective case series from 2000 to 2007. Sex Health. 2011;8(2):207-213.

6. Salhi B, Heilpern KL. Update on emerging infections: news from the Centers for Disease Control and Prevention. Symptomatic early neurosyphilis among HIV-positive men who have sex with men - four cities, United States, January 2002-June 2004. Ann Emerg Med. 2008;51(1):101-105.

7. Symptomatic early neurosyphilis among HIV-positive men who have sex with men - four cities, United States, January 2002-June 2004. MMWR Morb Mortal Wkly Rep. 2007;56(25):625-628. 
8. Zetola NM, Engelman J, Jensen TP, Klausner JD. Syphilis in the United States: an update for clinicians with an emphasis on HIV coinfection. Mayo Clin Proc. 2007;82(9):1091-1092.

9. O’Donnell JA, Emery CL. Neurosyphilis: a current review. Current Infect Dis Rep. 2005;7(4):277-284.

10. Brandt AM. Shadow on the land. In: No Magic Bullet: A Social History of Venereal Disease in the United States Since 1880. New York: Oxford University Press; 1987.

11. Marra CM. Update on neurosyphilis. Curr Infect Dis Rep. 2009;11(2): 127-134.

12. Knopman DS, DeKosky ST, Cummings JL, et al. Practice parameter: diagnosis of dementia (an evidence-based review). Report of the Quality Standards Subcommittee of the American Academy of Neurology. Neurology. 2001;56(9):1143-1153.

13. Lukehart SA. Syphilis. In: Fauci AS, Braunwald E, Kasper DL, et al, editors. Harrison's Principles of Internal Medicine. 17th ed. New York, NY: McGraw-Hill Medical; 2008: 1038-1046.
14. French P, Gomberg M, Janier M, Schmidt B, van Voorst Vader P, Young H. IUST. IUSTI: 2008 European guidelines on the management of syphilis. Int J STD AIDS. 2009;20(5):300-309.

15. Gilad R, LamplY, Blumstein G, Dan M. Neurosyphilis: the reemergence of an historical disease. Isr Med Assoc J. 2007;9(2):117-118.

16. Dourmishev LA, Dourmishev AL. Syphilis: uncommon presentations in adults. Clin Dermatol. 2005;23(6):555-564.

17. Tso MK, Koo K, Tso GY. Neurosyphilis in a non-HIV patient: more than a psychiatric concern. Mcgill J Med. 2008;11(2):160-163.

18. Workowski KA, Berman S; Centers for Disease Control and Prevention (CDC). Sexually Transmitted Diseases Treatment Guidelines, 2010. MMWR Recomm Rep. 2010;59(RR-12):1-110.

\section{Publish your work in this journal}

The International Medical Case Reports Journal is an international, peer-reviewed open-access journal publishing original case reports from all medical specialties. Previously unpublished medical posters are also accepted relating to any area of clinical or preclinical science. Submissions should not normally exceed 2,000 words or
4 published pages including figures, diagrams and references. The manuscript management system is completely online and includes a very quick and fair peer-review system, which is all easy to use. Visit http://www.dovepress.com/testimonials.php to read real quotes from published authors.

Submit your manuscript here: http://www.dovepress.com/international-medical-case-reports-journal-journal 